\title{
Autopercepção de saúde bucal e sua associação com fatores socioeconômicos- demográficos e condição de saúde bucal de idosos quilombolas
}

\author{
Self-perceived oral health and its association with socioeconomic-demographic factors and oral \\ health status in quilombola elderly \\ Autopercepción de la salud bucal y su asociación con factores socioeconómicos-demográficos y \\ estado de salud bucal de quilombolas ancianos
}

Recebido: 20/07/2021 | Revisado: 29/07/2021 | Aceito: 02/08/2021 | Publicado: 06/08/2021

\author{
Cledinaldo Lira Júnior \\ ORCID: https://orcid.org/0000-0002-2863-5513 \\ Universidade Estadual da Paraíba, Brasil \\ E-mail: junior_lira16@hotmail.com \\ Renata de Souza Coelho Soares \\ ORCID: https://orcid.org/0000-0001-5213-3698 \\ Universidade Estadual da Paraíba, Brasil \\ E-mail:drarenatacoelho@gmail.com \\ Tarciana Nobre de Menezes \\ ORCID: https://orcid.org/0000-0003-1784-3218 \\ Universidade Estadual da Paraíba, Brasil \\ E-mail: tnmenezes@yahoo.com.br
}

\begin{abstract}
Resumo
Objetivo: Avaliar a associação entre a autopercepção de saúde bucal e a condição de saúde bucal e fatores socioeconômico-demográficos de idosos quilombolas. Material e métodos: Trata-se de um estudo transversal realizado com 47 idosos quilombolas. A variável dependente foi a autopercepção de saúde bucal. As variáveis independentes foram as variáveis socioecônomico-demográficas e de saúde bucal. Foi realizado o Teste Qui-quadrado ou o Exato de Fisher, Mann-Whitney e Regressão de Poisson com variância robusta, todos com nível de significância de 5\%. Resultados: A média etária dos idosos foi 71 anos $(\mathrm{DP}= \pm 7,55)$. A análise bivariada mostrou associação da autopercepção de saúde bucal com a situação conjugal $(\mathrm{p}=0,020)$ e a necessidade de uso de prótese $(\mathrm{p}=0,003)$. A análise multivariada através da regressão de Poisson mostrou significância para a variável necessidade de uso de prótese $(\mathrm{p}<0,0001)$, logo, iIdosos com necessidade de uso de prótese apresentaram prevalência de autopercepção negativa de saúde bucal 52,3\% maior $(\mathrm{RP}=1,523$; IC95\%=0,109-0,612), quando comparados àqueles sem necessidade de prótese. Conclusão: De acordo com os achados do estudo o uso de prótese parece beneficiar autopercepção positiva de saúde bucal em idosos quilombolas.

Palavras-chave: Idosos; Saúde bucal; Índice de avaliação de saúde bucal geriátrica; Grupo africano de ancestrais continentais.
\end{abstract}

\begin{abstract}
Objective: to evaluate the association between self-perceived oral health and oral health status and socioeconomicdemographic factors in quilombola elderly people. Material and methods: This is a cross-sectional study carried out with 47 elderly quilombolas. Dependent variable of self-perceived oral health. The independent variables were the socioeconomic-demographic and oral health variables. The Chi-square or Fisher's Exact Test, Mann-Whitney and Poisson Regression with robust variance were performed, all with a significance level of 5\%. Results: The average age of the elderly was 71 years $(\mathrm{SD}= \pm 7.55)$. Bivariate analysis of the association of self-perceived oral health with marital status $(\mathrm{p}=0.020)$ and the need to use a prosthesis $(\mathrm{p}=0.003)$. A multivariate analysis using Poisson regression informing the significance for the variable need to use a prosthesis $(\mathrm{p}<0.0001)$, therefore, elderly people with a need for a prosthesis prevalence of negative self-perception of oral health $52.3 \%$ higher $(\mathrm{PR}=1,523 ; 95 \% \mathrm{CI}=$ 0.109 - 0.612), when compared to those without the need for a prosthesis. Conclusion: According to the findings of the study, the use of prosthesis seems to benefit from a positive self-perception of oral health in elderly quilombolas. Keywords: Elderly; Oral health; Geriatric oral health assessment index; African continental ancestry group.
\end{abstract}




\section{Resumen}

Objetivo: evaluar la asociación entre salud bucal autopercibida y estado de salud bucal y factores socioeconómicosdemográficos en quilombolas ancianos. Material y métodos: Se trata de un estudio transversal realizado con 47 quilombolas ancianos. Variable dependiente de la salud bucal autopercibida. Las variables independientes fueron las socioeconómicas-demográficas y de salud bucal. Se realizaron las pruebas de Chi-cuadrado o Exacta de Fisher, Regresión de Mann-Whitney y Poisson con varianza robusta, todas con un nivel de significancia del 5\%. Resultados: La edad promedio de los ancianos fue de 71 años $(\mathrm{DE}= \pm 7,55)$. Análisis bivariado de la asociación de la salud bucal autopercibida con el estado civil $(\mathrm{p}=0,020)$ y la necesidad de utilizar prótesis $(\mathrm{p}=0,003)$. Un análisis multivariado mediante regresión de Poisson informando la significancia de la variable necesidad de usar prótesis ( $\mathrm{p}<0,0001)$, por lo tanto, las personas mayores con necesidad de prótesis prevalencia de autopercepción negativa de la salud bucal $52,3 \%$ mayor $(\mathrm{RP}=1,523$; IC $95 \%=0,109-0,612)$, en comparación con aquellos sin necesidad de prótesis. Conclusión: De acuerdo con los hallazgos del estudio, el uso de prótesis parece beneficiar la autopercepción de salud bucal positiva en quilombolas ancianos.

Palabras clave: Anciano; Salud bucal; Índice de evaluación de la salud oral geriátrica; Grupo africano de ancestros continentales.

\section{Introdução}

O aumento do número de idosos é uma realidade que tem gerado novas demandas para os sistemas de saúde, uma vez que pessoas mais velhas têm necessidades específicas (Oliveira et al., 2016). Na área da Odontologia, estudos têm mostrado elevada prevalência de idosos apresentando problemas de saúde bucal como a perda dentária (Kassebaum et al., 2015; Klotz et al., 2017; Oliveira et al., 2016); cárie (López et al., 2017; Melo et al., 2016); e a doença periodontal (López et al., 2017).Essas doenças bucais afetam desproporcionalmente os indivíduos pobres e socialmente desfavorecidos da sociedade, uma vez que existe uma associação muito forte e consistente entre o nível socioeconômico e a ocorrência e gravidade das doenças bucais. Esta associação ocorre desde a primeira infância até a terceira idade (Peres et al., 2020).

Sob esta perspectiva de grupos vulneráveis, incluem-se os idosos de comunidades quilombolas, grupos étnico-raciais de trajetória histórica própria, dotados de relações territoriais específicas e com ancestralidade negra relacionada com a resistência ao opressivo momento histórico que ocorreu durante a escravidão no Brasil (Brasil, 2003). Estudos sobre a condição bucal de idosos quilombolas são escassos, ocasionando uma considerável lacuna de conhecimento acerca dessa população, especialmente no que se refere à condição bucal destes idosos.

Assim evidencia-se a necessidade de avaliar a associação de indicadores clínicos com indicadores psicológicos na avaliação de saúde, como é o caso da autopercepção de saúde bucal de idosos, verificada por meio do índice GOHAI (Geriatric Oral Health Assessment Index) (Atchison \& Dolan, 1990), cujos resultados podem auxiliar na implementação de políticas públicas em saúde que favoreçam os idosos dessas comunidades historicamente importantes.

A autopercepção de saúde considera as experiências prévias do indivíduo como participante de um contexto social, cultural e histórico e pode levar à procura por atendimento odontológico (Gilbert, 1994). Estudos utilizando o GOHAI como forma de avaliar a autopercepção de saúde bucal têm mostrado sua associação com a condição clínica de saúde oral (Esmeriz et al., 2012; Hernández-Palacios et al., 2015; Miranda et al., 2011; Mitri et al., 2020; Vasconcelos et al., 2012; Piuvezam \& de Lima, 2012). Essa informação é particularmente importante uma vez que a autopercepção de saúde é um indicador para saúde pública e está relacionado com a mortalidade (Idler \& Benyamini, 1997), morbidade e uso de serviço (Idler \& Benyamini, 1997; Tamayo-Fonseca et al., 2013).

Nessa perspectiva, considerando a lacuna que existe sobre a condição de saúde dos idosos quilombolas, este trabalho objetivou avaliar a associação entre a autopercepção de saúde bucal e a condição de saúde bucal, socioeconômico-demográfica em idosos quilombolas. 


\section{Metodologia}

Este é um estudo transversal, descritivo e analítico, realizado com idosos quilombolas da comunidade Caiana dos Crioulos, situada no município de Alagoa Grande, Paraíba, Brasil. A população do estudo foi constituída por todas as pessoas com 60 anos ou mais residentes nesta comunidade; 64 idosos segundo dados da Secretaria Municipal de Saúde de 2019 disponíveis no E-SUS. Após conferência destes dados junto ao Agente Comunitário de Saúde da região, ficou estabelecido o número de 55 idosos na comunidade.

Foram incluídos indivíduos de ambos os sexos, com 60 anos ou mais de idade, residentes na comunidade quilombola de Caiana dos Crioulos. Foram excluídos aqueles idosos que, por qualquer condição patológica, estavam impossibilitados de abrir a boca para realização de exames; idosos que apresentavam indicativo de declínio cognitivo; e idosos que estavam ausentes do quilombo durante o período de coleta de dados.

Foi realizado um estudo piloto com idosos moradores da comunidade quilombola Pedra D'água em Ingá, Paraíba, Brasil. A amostra do estudo piloto foi composta por 14 idosos, o que corresponde a aproximadamente $20 \%$ dos idosos residentes na comunidade quilombola Caiana dos Crioulos.

A calibração foi realizada por meio da comparação com um outro dentista especialista e com experiência em estudos epidemiológicos (padrão-ouro); observando a concordância inter-examinador comparando as análises do pesquisador com o padrão-ouro. Os valores de concordância foram obtidos através do coeficiente kappa de Cohen, sendo 0,86 para a concordância inter-examinador e 0,91 para a concordância intraexaminador.

Os quilombos são organizados em locais de difícil acesso e com terrenos bastante acidentados, o que inviabiliza o deslocamento de idosos para centros, nos quais pudessem ser realizados o estudo. A coleta dos dados foi realizada em visitas domiciliares, em momento único, no período de setembro a dezembro de 2019. As visitas foram agendadas previamente pelo Agente Comunitário de Saúde, havendo a possibilidade de remarcação da visita na impossibilidade de o idoso receber o pesquisador.

As variáveis socioeconômico-demográficas verificadas foram: gênero (feminino;masculino); faixa etária (60-70; mais de 70 anos); escolaridade (analfabeto; letrado); renda familiar(até um salário mínimo; mais de um salário mínimo); situação conjugal (sem cônjuge; com cônjuge); situação de coabitação (mora sozinho; não mora sozinho). As variáveis relativas à condição de saúde bucal foram: número de dentes ( $\geq 20$ dentes; $<20$ dentes); presença de prótese removível (não; sim); necessidade de prótese (não; sim); CPO-D e Índice Periodontal Comunitário (IPC) (saudável; sangramento gengival; presença de cálculo dentário; bolsa de 4-5mm e bolsas iguais ou superiores a 6mm).

A autopercepção de saúde bucal foi avaliada por meio do índice GOHAI desenvolvido para a população idosa (Atchison \& Dolan, 1990). O índice é composto por 13 perguntas que apresentam como opções de resposta: “sempre”, “às vezes" ou "nunca". Cada resposta recebe uma pontuação, sendo os valores de um ponto para "sempre", dois pontos para "as vezes" e três pontos para "nunca". O somatório das respostas classifica o idosos em: autopercepção de saúde bucal alta (entre 34 e 36 pontos), moderada (entre 33 e 30 pontos) e baixa (inferior a 30 pontos). Neste estudo a variável foi dicotomizada. Idosos que apresentaram pontuação do GOHAI menor que 34 foram classificados com autopercepção de saúde bucal "ruim” e idosos que apresentaram pontuação acima ou igual que 34 foram classificados com autopercepção "boa".

Os exames físicos foram realizados com o idoso sentado em uma cadeira comum, em local privado, sob iluminação natural. Foram utilizados equipamentos de proteção individual (EPI), espelho clínico plano nº4, sonda periodontal milimetrada OMS e espátulas de madeira. Observou-se a quantidade de dentes presentes na boca, a presença de alterações bucais em dentes e periodonto e se avaliou a presença/ou necessidade de próteses removíveis. 
Os dados foram tabulados em dupla entrada no software Microsoft Excel, cuja validação dos bancos foi verificada com a ferramenta "validate", do programa estatístico EpiInfo7.2. Foram realizadas análises bivariadas e multivariada no Statistical Package for the Social Sciences (SPSS para Windows versão 25.0; IBM Inc., Armonk, NY, EUA).

As análises bivariadas foram realizadas por meio do Teste do Qui-quadrado ou Exato de Fisher para mensurar a magnitude da associação entre variáveis explicativas e desfecho, razões de prevalências (RP) e seus respectivos intervalos de confiança (IC 95\%). Para testar a diferença entre a mediana dos grupos da variável GOHAI, foi realizado o teste MannWhitney. Os resultados foram expressos em tabelas, tendo sido fixado um nível de significância de 5\% para todos os testes.

Para obter estimativas da Razão de Prevalência foi realizada uma análise multivariada, por meio do modelo de Regressão de Poisson com variância robusta. Foram selecionadas, para a análise multivariada, as variáveis que na associação com o desfecho na análise bivariada apresentaram valor de $\mathrm{p}<0,20$. No modelo final foram mantidas aquelas variáveis com associação significante no nível de $\mathrm{p}<0,05$. A adequação do modelo final foi observada através do teste de Omnibus.

A pesquisa foi realizada em conformidade com a Resolução 466/2012 do Conselho Nacional de Saúde do Ministério da Saúde que regulamenta pesquisas com seres humanos. A pesquisa maior, da qual esta pesquisa faz parte, foi aprovada pelo Comitê de Ética em Pesquisa da Universidade Estadual da Paraíba (parecer número 3.459.657).

\section{Resultados}

Participaram do estudo 47 idosos quilombolas, de ambos os sexos, residentes no quilombo Caiana dos Crioulos, município de Alagoa Grande, Paraíba, Brasil. A idade variou de 60 a 92 anos, com média de 71 anos (DP = +/- 7, 55). Entre os idosos quilombolas, observou-se que a maioria apresentava 60 a 70 anos $(53,2 \%)$, era do sexo feminino $(68,1 \%)$, apresentava cônjuge $(57,4 \%)$, tinha renda familiar menor ou igual a um salário mínimo $(85,1 \%)$, era analfabeta $(55,3 \%)$ e não vivia sozinha em suas residências $(80,9 \%)$ (Tabela 1$)$.

A média da pontuação do GOHAI foi 30,77 ( $\mathrm{DP}=+$ +- 5,09). Este valor, segundo os critérios do índice, classifica a autopercepção da saúde bucal como moderada. Entre os idosos estudados, 55,3\% autoperceberam a saúde bucal como "ruim". Na Tabela 1 é possível observar que foi verificada associação significante entre a autopercepção de saúde bucal e a situação conjugal ( $\mathrm{p}=0,020)$ e entre a autopercepção de saúde bucal e a necessidade de prótese ( $\mathrm{p}=0,003)$.

O CPO-D médio para a população foi $25,60(+/-8,5)$, apresentando o componente "perdido" como responsável por 96,7\% da composição total do índice. Logo, a prevalência de idosos com menos de 20 dentes foi alta (87,3\%), sendo 40,4\% deles totalmente edêntulos

Quando da aplicação do CPI, não foi possível realizar a análise da condição periodontal em 24 idosos $(51,1 \%)$ devido à quantidade de sextantes excluídos. Dentre os idosos avaliados, a prevalência dos maiores escores identificados foi a seguinte: 17\% apresentaram o cálculo dentário como maior escore, 14,9\% apresentaram bolsas periodontais rasas e 17\% apresentaram bolsas profundas. A limitação imposta pela quantidade de idosos excluídos no exame impediram a realização do uso desta variável para testes mais robustos. 
Tabela 1: Análise bivariada da autopercepção de saúde bucal com as variáveis socioeconômico-demográficas e de condição de saúde bucal. Alagoa Grande, Paraíba, Brasil, 2019.

\begin{tabular}{|c|c|c|c|c|c|c|c|}
\hline \multirow[b]{2}{*}{ Variáveissocieoeconômico-demográficas e de saúde bucal } & \multicolumn{7}{|c|}{ Autopercepção de saúde bucal } \\
\hline & $\mathbf{N}$ & $\%$ & Ruim & $(\%)$ & Boa & $(\%)$ & $\mathbf{p}$ \\
\hline Total de idosos & 47 & 100 & 26 & 55,3 & 21 & 44,7 & \\
\hline \multicolumn{8}{|l|}{ Faixa etária } \\
\hline $60-70$ anos & 25 & 53,2 & 12 & 25,5 & 13 & 27,7 & \multirow{2}{*}{$0,282 *$} \\
\hline$>70$ anos & 22 & 46,8 & 14 & 29,8 & 8 & 17 & \\
\hline \multicolumn{8}{|l|}{ Gênero } \\
\hline Feminino & 32 & 68,1 & 18 & 38.3 & 14 & 29,8 & \multirow{2}{*}{$0,851^{*}$} \\
\hline Masculino & 15 & 31,9 & 8 & 17 & 7 & 14,9 & \\
\hline \multicolumn{8}{|l|}{ Situação Conjugal } \\
\hline Sem cônjuge & 20 & 42,6 & 15 & 31,9 & 5 & 10,6 & \multirow{2}{*}{$0,020 *$} \\
\hline Com cônjuge & 27 & 57,4 & 11 & 23,4 & 16 & 34,6 & \\
\hline \multicolumn{8}{|l|}{ Renda Familiar } \\
\hline Até 1 salário mínimo**** & 40 & 85,1 & 22 & 46,8 & 18 & 38,3 & \multirow{2}{*}{$1,000 * *$} \\
\hline Mais de 1 salário mínimo**** & 7 & 14,9 & 4 & 8,5 & 3 & 6,4 & \\
\hline \multicolumn{8}{|l|}{ Escolaridade } \\
\hline Analfabeto & 26 & 55,3 & 16 & 34 & 10 & 21,3 & \multirow{2}{*}{$0,340 *$} \\
\hline Letrado & 21 & 44,7 & 10 & 21,3 & 11 & 23,4 & \\
\hline \multicolumn{8}{|l|}{ Situação de coabitação } \\
\hline Mora sozinho & 9 & 19,1 & 7 & 17,7 & 2 & 4,3 & \multirow{2}{*}{$0,160 * *$} \\
\hline Não mora sozinho & 38 & 80,9 & 19 & 40,4 & 19 & 40,4 & \\
\hline \multicolumn{8}{|l|}{ Número de dentes } \\
\hline Maior ou igual a 20 & 6 & 12,8 & 2 & 4,3 & 4 & 8,5 & \multirow{2}{*}{$0,386 * *$} \\
\hline Menos que 20 & 41 & 87,2 & 24 & 51,1 & 17 & 36,2 & \\
\hline \multicolumn{8}{|l|}{ Presença de prótese removível } \\
\hline Não & 32 & 68,1 & 20 & 42,6 & 12 & 25,5 & \multirow{2}{*}{$0,148^{*}$} \\
\hline Sim & 15 & 31,9 & 6 & 12,8 & 9 & 19,1 & \\
\hline \multicolumn{8}{|l|}{ Necessidade de prótese } \\
\hline Não & 10 & 21,3 & 1 & 2,1 & 9 & 19,1 & \multirow{2}{*}{$0,003 * *$} \\
\hline Sim & 37 & 78,7 & 25 & 53,2 & 12 & 25,5 & \\
\hline
\end{tabular}


* Qui-quadrado

**Exato de Fisher

***Mann-Whitney

**** Salário mínimo em 2019: 998,00 R \$

Fonte: Dados da pesquisa.

Na Tabela 2 é apresentada a análise de regressão de Poisson com variância robusta, quando foram utilizadas as variáveis com $p<0,20$ na análise bivariada: "situação conjugal" ( $p=0,020)$, "situação de coabitação" ( $p=0,160)$, "presença de prótese removível" ( $p=0,148)$, “necessidade de prótese" $(p=0,003)$ e "CPO-D” $(p=0,197)$.

Tabela 2: Análise multivariada para a autopercepção de saúde bucal ruim e variáveis socioeconômico-demográficas e de saúde bucal em idosos quilombolas. Alagoa Grande, Paraíba, Brasil, 2019.

\begin{tabular}{|c|c|c|}
\hline Variáveis socioeconômicas e de saúde bucal & RP bruta (IC 95\%) & $\mathbf{P}$ \\
\hline \multicolumn{3}{|l|}{ Situação conjugal } \\
\hline Com cônjuge & 1 & \multirow{2}{*}{0,188} \\
\hline Sem cônjuge & $1,145(0,936-1,400)$ & \\
\hline \multicolumn{3}{|l|}{ Situação de coabitação } \\
\hline Não mora sozinho & 1 & \multirow{2}{*}{0,758} \\
\hline Mora sozinho & $1,033(0,841-1,268)$ & \\
\hline \multicolumn{3}{|l|}{ Presença de prótese removível } \\
\hline Não & 1 & \multirow{2}{*}{0,721} \\
\hline Sim & $0,964(0,178-1,178)$ & \\
\hline \multicolumn{3}{|l|}{ Necessidade de prótese } \\
\hline Não & 1 & \multirow{2}{*}{$0,004 *$} \\
\hline Sim & $1,396(1,116-1,747)$ & \\
\hline CPO-D & $1,009(0,998-1,019)$ & 0,106 \\
\hline
\end{tabular}

Fonte: Dados da pesquisa.

Após o ajuste do modelo, apenas a variável necessidade de prótese mostrou-se significante. Com isso, observa-se que idosos com necessidade de prótese apresentaram prevalência de autopercepção de saúde bucal ruim 52,3\% maior $(\mathrm{RP}=1,523$; IC95\%= 1,258 - 1,845), quando comparados àqueles sem necessidade de prótese (Tabela 3). 
Tabela 3: Análise multivariada para a autopercepção bucal ruim em idosos quilombolas ajustada para a variável "necessidade de prótese". Alagoa Grande, Paraíba, Brasil, 2019.

\begin{tabular}{lcccc}
\hline Variável & $\begin{array}{c}\text { RP bruta } \\
\text { (IC 95\%) }\end{array}$ & P & $\begin{array}{c}\text { RP ajustada } \\
\text { (IC 95\%) }\end{array}$ & P \\
\hline Necessidade de prótese & & & & \\
\hline Não & 1 & 0,004 & 1 & 0,000 \\
\hline Sim & 1,396 & & 1,523 \\
\end{tabular}

Fonte: Autores (2021).

\section{Discussão}

Os resultados deste estudo indicam associação entre a necessidade protética e a autopercepção de saúde bucal ruim entre os idosos quilombolas avaliados.

A média do GOHAI dos idosos foi 30,77, média semelhante à encontrada em outros estudos realizados com idosos (Carvalho et al., 2016; Esmeriz et al., 2012; Miranda et al., 2011; Mitri et al., 2020). Neste trabalho, as categorias propostas pelos idealizadores do GOHAI foram recategorizadas em "boa" ou "ruim", o que mostrou elevada prevalência de idosos com autopercepção de saúde bucal ruim. Essa elevada taxa foi observada por Miranda e Silveira (2011) (Miranda et al., 2011) (68,2\%), assim como por Mitri, Sayegh e Boulos (2020) (Mitri et al., 2020) (51,6\%,), em estudos com idosos.

A autopercepção de saúde bucal ruim encontrada neste estudo foi coerente com a condição bucal observada a partir do exame clínico, que evidenciou número elevado de dentes perdidos e necessidade de reabilitação protética. Ekanayke \& Perera (2005) (Ekanayke \& Perera, 2005) verificaram em seu estudo autopercepção de saúde bucal boa nos idosos com mais de 20 dentes. Sobre isto Steele et al (2004) (Steele et al., 2004) afirmaram que o número de dentes presentes é um importante determinante da percepção subjetiva da saúde bucal.

Informações da análise bivariada mostraram haver associação entre autopercepção de saúde bucal e situação conjugal. O estudo de Miranda et al. (2011) (Miranda et al., 2011) mostrou que idosos que não eram casados tinham uma chance 2,06 vezes maior de autoperceberem sua condição bucal como ruim, quando comparados aos idosos casados. Carvalho et al., (2016) (Carvalho et al., 2016) descreveram que os idosos solteiros apresentaram maior probabilidade de autopercepção de saúde bucal ruim que aqueles casados ou em união estável.

A insegurança de não ter com quem contar contribui para a pior avaliação da saúde (Nunes et al., 2012). Nesta perspectiva, os idosos que apresentam cônjuges parecem ter um apoio social que pode contribuir com a melhoria da percepção acerca da sua saúde bucal, ao passo que aqueles sem cônjuges, apesar de por vezes estarem cercados por outras pessoas, não teriam a vantagem de ter alguém íntimo ao seu lado. A associação com a situação conjugal deste trabalho sugere que a situação conjugal avalia a importância do apoio social e familiar para o desfecho, assim como tem a especificidade de avaliar a importância do cônjuge para a uma boa autopercepção de saúde bucal.

No contexto da importância do apoio social, apesar de neste estudo não ter sido verificada a associação entre a autopercepção de saúde bucal e a situação de coabitação em idosos quilombolas, esta relação precisa ser considerada em um cenário de avaliação global da saúde do idoso. Idosos que moram sozinhos devem ser apoiados pelo Estado e pela comunidade, e os serviços de assistência precisam estar preparados para o cuidado destas pessoas (Teixeira et al., 2016). 
Quanto à análise multivariada, foi encontrado associação entre a autopercepção da saúde bucal e a necessidade de prótese. Outros estudos realizados com idosos também têm verificado resultados semelhantes (Klotz et al., 2017; Kressin et al., 1997; Miranda et al., 2011; Mitri et al., 2020; Vale et al., 2016; Vasconcelos et al., 2012).

Miranda et al. (2011) (Miranda et al., 2011) verificaram que idosos que necessitavam de prótese apresentaram chance 10,87 vezes maior de autoperceber sua condição como "ruim" em comparação àqueles que não necessitavam. Klotz et al., 2017 (Klotz et al., 2017) mostraram um risco 6,5 vezes maior de autopercepção bucal ruim, entre os idosos que necessitavam de próteses totais e um risco 2,5 vezes maior de autopercepção de saúde bucal ruim entre idosos com necessidades de outros tipos de próteses. Mitri et al. (2020) (Mitri et al., 2020) encontraram que não usar próteses ou fazer uso de peças mal adaptadas aumentaram mais de dez vezes a chance de autopercepção ruim em comparação com aqueles cujas próteses atendiam às suas necessidades.

O uso de prótese possibilita melhorar a mastigação, fonação, deglutição e a autoestima do idoso com perdas dentárias (Kurahashi et al., 2015), além de seu uso estar associado à melhoria da autopercepção de saúde bucal (Dable et al., 2013; Ha et al., 2012). Desta maneira, idosos que necessitam de próteses, além de estarem suscetíveis aos fatores negativos impostos pela ausência da reabilitação, têm a autopercepção de saúde bucal prejudicada, uma vez que a autoavaliação negativa está relacionada à necessidade de reabilitação protética parcial ou total (Vilela et al., 2013).

Em se tratando de idosos quilombolas, as limitações impostas pela ausência dentária são ainda mais preocupantes, uma vez que idosos pretos possuem mais dificuldade de acesso a bens e serviços (Silva et al., 2019). Essa dificuldade impossibilita a reabilitação através de próteses, comprometendo a saúde e diminuindo a qualidade de vida destas pessoas. Souza et al. (2012) (Souza et al., 2012), fazendo uso dos dados da Pesquisa Nacional de Saúde Bucal Brasileira, mostraram que a raça é um fator limitante na utilização dos serviços odontológicos, atentando para o fato de que idosos pretos apresentarem maior dificuldade no acesso aos serviços de saúde bucal.

Apesar de neste estudo não ter sido encontrada associação entre autopercepção de saúde bucal ruim e o número de dentes, há que se considerar a elevada prevalência de edentulismo, tendo em vista que a perda dentária constitui um problema frequente que está fortemente associado a um impacto negativo na autopercepção de saúde bucal e na qualidade de vida relacionada à saúde bucal (Gerritsen et al., 2010). Além disso, a ausência de associações com outras variáveis socioeconômicodemográficas deste estudo, como a renda e a escolaridade, pode ter ocorrido devido à homogeneidade destas informações entre os idosos, o que demandou diminuição do poder da análise estatística.

As comunidades quilombolas compartilham aspectos de vulnerabilidade com outras populações rurais e com a população negra brasileira, compartilhando de situação de fragilidade, tendo menor acesso a serviços de saúde (Kassouf, 2005), maior risco de agravos à saúde bucal (de Campos Mello et al., n.d.), além de pior autopercepção de saúde, tanto geral como bucal (Moura et al., 2014). Os resultados deste estudo são representativos do grupo de idosos de Caiana dos Crioulos, o que garante sua validade interna. Entretanto, devido às similaridades compartilhadas por essas pessoas, poderiam ser extrapolados para idosos de outros quilombos rurais, podendo, ainda, serem utilizados como aporte para nortear as políticas de saúde dos idosos quilombolas, ao constituir evidência científica acerca da saúde destas populações.

\section{Conclusão}

Este estudo mostrou a existência de associação entre e autopercepção de saúde bucal ruim e necessidade de prótese entre os idosos quilombolas. Nesta perspectiva, é razoável atentar para o fato que a prevenção da perda dentária e o tratamento reabilitador com próteses parecem ser importantes para a manutenção de uma boa autopercepção de saúde bucal, melhorando a qualidade de vida de idosos quilombolas. 
Partindo da premissa da dificuldade de acesso aos serviços de saúde por idosos quilombolas, é desejável a elaboração de estratégias de atenção à saúde e o estabelecimento de políticas públicas que possibilitem o acesso equitativo destes indivíduos aos serviços de saúde (bucal e geral) nas comunidades quilombolas, respeitando os valores comunitários e ainda promovendo a participação social no seu processo de cuidado.

A realização de estudos longitudinais são encorajados, de forma a evidenciarem a relação entre a condição de saúde bucal e a autopercepção de saúde bucal, bem como elucidarem outros problemas de saúde bucal existentes entre os idosos quilombolas.

\section{Referências}

Atchison, K. A., \& Dolan, T. A. (1990). Development of the Geriatric Oral Health Assessment Index. In Journal of Dental Education. 54(11), 680-7. https://doi.org/10.1002/j.0022-0337.1990.54.11.tb02481.x.

Brasil, Decreto $N^{\circ}$ 4.887, de 20 de novembro de 2003 (2003), Regulamenta o procedimento para identificação, reconhecimento, delimitação, demarcação e titulação das terras ocupadas por remanescentes das comunidades dos quilombos de que trata o art. 68 do Ato das Disposições Constitucionais Transitórias. http://www.planalto.gov.br/ccivil_03/decreto/2003/d4887.htm.

Carvalho, C., Manso, A. C., Escoval, A., Salvado, F., \& Nunes, C. (2016). Self-perception of oral health in older adults from an urban population in Lisbon, Portugal. Revista de Saude Publica. 50(53), 1-9. https://doi.org/10.1590/S1518-8787.2016050006311.

Dable, R. A., Nazirkar, G. S., Singh, S. B., \& Wasnik, P. B. (2013). Assessment of Oral Health Related Quality of Life Among Completely Edentulous Patients in Western India by Using GOHAI. Journal of Clinical and Diagnostic Research: JCDR,.7(9), 2063-7. https://doi.org/10.7860/JCDR/2013/6377.3406.

de Campos Mello, T. R., Antunes, J. L. F., \& Waldman, E. A. (2005). Áreas Rurais: Pólos de Concentração de Agravos à Saúde Bucal? Arquivos de Medicina. 19(1-2), 67-74. http://www.scielo.mec.pt/pdf/am/v19n1-2/v19n1a10.pdf.

Ekanayke, L., \& Perera, I. (2005). Factors associated with perceived oral health status in older individuals. International Dental Journal. 55(1), 31-37. https://doi.org/10.1111/j.1875-595x.2005.tb00029.x.

Esmeriz, C. E. C., Meneghim, M. C., \& Ambrosano, G. M. B. (2012). Self-perception of oral health in non-institutionalised elderly of Piracicaba city, Brazil. Gerodontology, 29(2), e281-e289. https://doi.org/10.1111/j.1741-2358.2011.00464.x.

Gerritsen, A. E., Allen, P. F., Witter, D. J., Bronkhorst, E. M., \& Creugers, N. H. J. (2010). Tooth loss and oral health-related quality of life: a systematic review and meta-analysis. Health and Quality of Life Outcomes. 8(126), 1-11. https://doi.org/10.1186/1477-7525-8-126.

Gilbert, L. (1994). Social factors and self-assessed oral health in South Africa. Community Dentistry and Oral Epidemiology. 22(1), 47-51. https://doi.org/10.1111/j.1600-0528.1994.tb01568.x.

Ha, J. E., Heo, Y. J., Jin, B. H., Paik, D. I., \& Bae, K. H. (2012). The impact of the National Denture Service on oral health-related quality of life among poor elders. Journal of Oral Rehabilitation. 39(8), 600-7. https://doi.org/10.1111/j.1365-2842.2012.02296.x.

Hernández-Palacios, R. D., Ramírez-Amador, V., Jarillo-Soto, E. C., Irigoyen-Camacho, M. E., \& Mendoza-Núñez, V. M. (2015). Relationship between gender, income and education and self-perceived oral health among elderly Mexicans. An exploratory study. Ciencia \& Saude Coletiva. 20(4), 997-1004. https://doi.org/10.1590/1413-81232015204.00702014.

Idler, E. L., \& Benyamini, Y. (1997). Self-rated health and mortality: a review of twenty-seven community studies. Journal of Health and Social Behavior. 38(1), 21-37. https://www.ncbi.nlm.nih.gov/pubmed/9097506.

Kassebaum, N. J., Bernabé, E., Dahiya, M., Bhandari, B., Murray, C. J. L., \& Marcenes, W. (2015). Global burden of untreated caries: a systematic review and metaregression. Journal of Dental Research. 94(5), 650-8. https://doi.org/10.1177/0022034515573272.

Kassouf, A. L. (2005). Acesso aos serviços de saúde nas áreas urbana e rural do Brasil. In Revista de Economia e Sociologia Rural. 43(1), 29-44. https://doi.org/10.1590/s0103-20032005000100002.

Klotz, A.-L., Hassel, A. J., Schröder, J., Rammelsberg, P., \& Zenthöfer, A. (2017). Oral health-related quality of life and prosthetic status of nursing home residents with or without dementia. In Clinical Interventions in Aging. 12(1), 659-65. https://doi.org/10.2147/cia.s125128.

Kressin, N. R., Atchison, K. A., \& Miller, D. R. (1997). Comparing the impact of oral disease in two populations of older adults: application of the geriatric oral health assessment index. Journal of Public Health Dentistry. 57(4), 224-232. https://doi.org/10.1111/j.1752-7325.1997.tb02979.x.

Kurahashi, M., Kondo, H., Iinuma, M., Tamura, Y., Chen, H., \& Kubo, K.-Y. (2015). Tooth Loss Early in Life Accelerates Age-Related Bone Deterioration in Mice. In The Tohoku Journal of Experimental Medicine. 235(1) 29-37. https://doi.org/10.1620/tjem.235.29.

López, R., Smith, P. C., Göstemeyer, G., \& Schwendicke, F. (2017). Ageing, dental caries and periodontal diseases. In Journal of Clinical Periodontology. 44(1), S145-52. https://doi.org/10.1111/jcpe.12683.

Melo, L. A. de, Sousa, M. de M., Medeiros, A. K. B. de, Carreiro, A. da F. P., \& Lima, K. C. de. (2016). Factors associated with negative self-perception of oral health among institutionalized elderly. Ciencia \& saude coletiva. 21(11), 3339-46. https://doi.org/10.1590/1413-812320152111.08802015. 
Miranda, L. de P., de Paula Miranda, L., Silveira, M. F., de Paula Miranda, L., \& Bonan, P. R. F. (2011). Autopercepção das condições bucais em uma população de idosos da cidade de Montes Claros, Minas Gerais, Brasil. In Revista Brasileira de Geriatria e Gerontologia. 14(2), 251-69). https://doi.org/10.1590/s1809-98232011000200007.

Mitri, R., Fakhoury Sayegh, N., \& Boulos, C. (2020). Factors associated with oral health-related quality of life among Lebanese community-dwelling elderly. Gerodontology, 37(2), 200-7. https://doi.org/10.1111/ger.12463.

Moura, C., Gusmão, E. S., Santillo, P. M. H., de Souza Coelho Soares, R., \& Cimões, R. (2014). Autoavaliação da saúde bucal e fatores associados entre adultos em áreas de assentamento rural, Estado de Pernambuco, Brasil. In Cadernos de Saúde Pública, 30(3), 611-22). https://doi.org/10.1590/0102$311 \times 00117012$

Nunes, A. P. N., Barreto, S. M., \& Gonçalves, L. G. (2012). Relações sociais e autopercepção da saúde: projeto envelhecimento e saúde. In Revista Brasileira de Epidemiologia. 15(2), 415-28). https://doi.org/10.1590/s1415-790x2012000200019.

Oliveira, M. R. de, de Oliveira, M. R., Veras, R. P., de Albuquerque Cordeiro, H., \& Pasinato, M. T. (2016). A mudança de modelo assistencial de cuidado ao idoso na Saúde Suplementar: identificação de seus pontos-chave e obstáculos para implementação. In Physis: Revista de Saúde Coletiva. 26(4), 1383-94. https://doi.org/10.1590/s0103-73312016000400016.

Peres, M. A., Daly, B., Guarnizo-Herreño, C. C., Benzian, H., \& Watt, R. G. (2020). Oral diseases: a global public health challenge - Authors' reply [Review of Oral diseases: a global public health challenge - Authors' reply]. The Lancet, 395(10219), 186-7. https://doi.org/10.1016/S0140-6736(19)32997-6.

Piuvezam, G., \& de Lima, K. C. (2012). Self-perceived oral health status in institutionalized elderly in Brazil. Archives of Gerontology and Geriatrics, 55(1), 5-11. https://doi.org/10.1016/j.archger.2011.04.017.

Silva, A. da, Rosa, T. E. da C., Batista, L. E., Kalckmann, S., Louvison, M. C. P., Teixeira, D. S. da C., \& Lebrão, M. L. (2019). Iniquidades raciais e envelhecimento:análise da coorte 2010 do Estudo Saúde,Bem-Estar e Envelhecimento (SABE). Revista brasileira de epidemiologia = Brazilian journal of epidemiology. 21(2), 1-14. https://doi.org/10.1590/1980-549720180004.supl.2.

Souza, E. H. A. de, de Souza, E. H. A., de Oliveira, P. A. P., Paegle, A. C., \& de Goes, P. S. A. (2012). Raça e o uso dos serviços de saúde bucal por idosos. In Ciência \& Saúde Coletiva. 17(8), 2063-70). https://doi.org/10.1590/s1413-81232012000800017.

Steele, J. G., Sanders, A. E., Slade, G. D., Allen, P. F., Lahti, S., Nuttall, N., \& Spencer, A. J. (2004). How do age and tooth loss affect oral health impacts and quality of life? A study comparing two national samples. Community Dentistry and Oral Epidemiology. 32(2), 107-114. https://doi.org/10.1111/j.03015661.2004.00131.x.

Tamayo-Fonseca, N., Quesada, J. A., Nolasco, A., Melchor, I., Moncho, J., Pereyra-Zamora, P., López, R., Calabuig, J., \& Barber, X. (2013). Self-rated health and mortality: a follow-up study of a Spanish population. Public Health. 127(12), 1097-104. https://doi.org/10.1016/j.puhe.2013.09.003.

Teixeira, D. S. da C., da Cruz Teixeira, D. S., Frazão, P., Alencar, G. P., Baquero, O. S., Narvai, P. C., Lebrão, M. L., \& de Oliveira Duarte, Y. A. (2016). Estudo prospectivo da perda dentária em uma coorte de idosos dentados. In Cadernos de Saúde Pública. 32(8), 1-11. https://doi.org/10.1590/0102$311 \times 00017215$

Vale, M. J. L., Flório, F. M., Zanin, L., Ambrosano, G. M. B., \& Santos, R. B. (2016). Autopercepção de idosos de Teresina-PI sobre saúde bucal e fatores associados. Arquivos em Odontologia, 52(1), 46-56. https://periodicos.ufmg.br/index.php/arquivosemodontologia/article/view/3688.

Vasconcelos, L. C. A. de, Prado Júnior, R. R., Teles, J. B. M., \& Mendes, R. F. (2012). [Self-perceived oral health among elderly individuals in a mediumsized city in Northeast Brazil]. Cadernos de saude publica, 28(6), 1101-10. https://doi.org/10.1590/s0102-311x2012000600009.

Vilela, E. A., de Barros Lima Martins, A. M. E., Barreto, S. M., Vargas, A. M. D., \& Ferreira, R. C. (2013). Association between self-rated oral appearance and the need for dental prostheses among elderly Brazilians. In Brazilian Oral Research. 27(3), 203-10). https://doi.org/10.1590/s1806-83242013005000010 